\title{
Spin Flavor Oscillation of Neutrinos in Rotating Gravitational Fields and Their Effects on Pulsar Kicks
}

\author{
G. Lambiase \\ Dipartimento di Fisica E.R. Caianiello, Universita' di Salerno, 84081 - Baronissi (SA), Italy, \\ and \\ INFN, Gruppo collegato di Salerno, Italy
}

Received on 19 December, 2004

\begin{abstract}
The origin of high velocities of pulsars is studied by considering the spin-flip conversion of neutrinos propagating in a gravitational field of a protoneutron star. For a rotating gravitational source (such as pulsars) with angular velocity, one finds that the spin connections (entering in the Dirac equation written in curved space time) induce an additional contribution to neutrino energy which is proportional to $\boldsymbol{\omega} \cdot \mathbf{p}$, with $\mathbf{p}$ the neutrino momentum. Such a coupling (spin-gravity coupling) can be responsible of pulsar kicks being the asymmetry of the neutrino emission generated by the relative orientation of the neutrino momentum $\mathbf{p}$ with respect to the angular velocity $\boldsymbol{\omega}$. As a consequence, the mechanism suggests that the motion of pulsars is correlated to their angular velocity $\boldsymbol{\omega}$. In this work we consider neutrinos propagating orthogonally to the magnetic field. The fractional asymmetry turns out to be independent on the magnetic field of the nascent protostar, and is only related to the angular velocity $(\Delta p / p \sim \omega)$. As in the usual approaches, spin flip conversion is generated via the coupling of the neutrino magnetic momentum with the magnetic field. For our estimations, we use the large non-standard neutrino magnetic momentum provided by astrophysical and cosmological constraints, $\mu_{\nu} \sim 10^{-11} \mu_{B}$. - The connection with recent observations and statistical analysis is also discussed.
\end{abstract}

\section{Introduction}

A discussed and unsolved issue of the modern astrophysics is the origin of the pulsar velocity, i.e. the high proper velocities of pulsars as compared with the surrounding stars. Their three-dimensional galactic speed runs, in fact, from $450 \pm 90 \mathrm{Km} / \mathrm{sec}$ up to a maximum of about $1000 \mathrm{Km} / \mathrm{sec}[1]$. This peculiarity immediately has suggested that nascent pulsars receive an impulse (kick) during their formation. The gravitational binding energy $\left(3 \times 10^{53} \mathrm{erg}\right)$ is, after the supernova collapse of a massive star, carried out by outgoing neutrinos (about $99 \%$ ). An anisotropy of $\sim 1 \%$ of the momenta distribution of the outgoing neutrinos would then suffice to account for the neutron star recoil of $300 \mathrm{Km} / \mathrm{sec}$.

An interesting mechanism to generate the pulsar velocity has been recently proposed by Kusenko and Segré (KS) [2]. It involves the physics of neutrino oscillation in presence of an intense magnetic field. Let us recall the basic idea. The neutrinosphere is defined as the surface from which neutrinos may escape from the protostars. In particular, electron neutrinos $\nu_{e}$ are emitted from a surface which is located at a distance from the center greater than the surfaces corresponding to muon/tau $\left(\nu_{\mu, \tau}\right)$ neutrinospehres. Under suitable conditions, a resonant oscillation $\nu_{e} \rightarrow \nu_{\mu, \tau}$ can occur between the $\nu_{e}$ and $\nu_{\mu, \tau}$ neutrinospheres. Neutrinos $\nu_{\mu, \tau}$ generate via oscillations can escape from the protostar being outside of their neutrinosphere, with the ensuing that the "surface of the resonance" acts as an "effective $\nu_{\mu, \tau}$-neutrinosphere". The presence of a magnetic field may distort the effective surface of resonance and the energy flux turns out to be generated anisotropically. In the KS mechanism [2], the responsible for the neutrino emission anisotropy is the polarization of the medium due to the magnetic field $\mathbf{B}$. The usual MSW resonance conditions turn out to be, in fact, modified by the term (first derived by D'Olivo, Nieves and Pal) [3]

$$
\frac{e G_{F}}{\sqrt{2}}\left(\frac{3 n_{e}}{\pi^{4}}\right)^{1 / 3} \mathbf{B} \cdot \hat{\mathbf{p}}
$$

where $\hat{\mathbf{p}}=\mathbf{p} / p, \mathbf{p}$ is the neutrino momentum, $e$ is the electric charge, $G_{F}$ is the Fermi constant, and $n_{e}$ is the electron density. The KS mechanism has been also studied for active-sterile neutrino oscillations (sterile neutrinos may have a small mixing angle with the ordinary neutrinos) [4, 5]. Papers dealing with the origin of pulsar kicks can be found in [6-25].

In this paper is discussed the possibility that spin flavor conversion of neutrinos propagating in a gravitational field generated by a rotating source may generate the observed pulsar kicks [24] (the role of the angular velocity on pulsar kicks has been also studied, although in a different context, by Mosquera Cuesta [18]). The gravitational field affects, as we will see, the resonance conversion of left-handed neutrinos into right handed neutrinos, the latter being sterile can escape from the neutrinosphere, hence from the protoneutron star. In particular, the modification to the resonance condition is induced by spin connections which enter in the Dirac 
equation in curved spacetimes. They give rise to a coupling term $\sim \boldsymbol{\omega} \cdot \mathbf{p}$, where $\boldsymbol{\omega}$ is the angular velocity of the gravitational source. The relative orientation of neutrino momenta with respect to the angular velocity determines an asymmetry in the neutrino emission, hence may generate pulsar kicks.

The paper is organized as follows. In Sect. 2 we review the Dirac equation in curved space-times. Here we see that, owing to the breakdown of the spherical symmetry generated by the angular velocity of the gravitational source, spin connections are non vanishing and are proportional to the chiral operator $\gamma^{5}$. Sect. 3 and Sect. 4 are devoted, respectively, to briefly recall the main features of matter induced effective potential and the role and intensity of magnetic fields in astrophysical systems. In Sect. 5 the fractional asymmetry is computed. Here we also discuss the resonance and adiabatic conditions, as well as the spin flip probability that left-handed neutrinos transform in right-handed neutrinos. Conclusions are drawn in Sect. 6

\section{Dirac Equation in Curved Space Time}

The phase of neutrinos propagating in a curved background is generalized as $[26,27]$

$$
\left|\psi_{f}(\lambda)\right\rangle=\sum_{j} U_{f j} e^{i \int_{\lambda_{0}}^{\lambda} P \cdot p_{\text {null }} d \lambda^{\prime}}\left|\nu_{j}\right\rangle,
$$

where $f$ is the flavor index and $j$ the mass index. $U_{f j}$ are the matrix elements transforming flavor and mass bases

$$
U=\left(\begin{array}{cc}
\cos \theta & \sin \theta \\
-\sin \theta & \cos \theta
\end{array}\right)
$$

(in what follows we shall consider the neutrino mixing for two flavors). Besides,

$$
P \cdot p_{\text {null }}=P_{\mu} p_{\text {null }}^{\mu},
$$

where $P_{\mu}$ is the four-momentum operator generating spacetime translation of the eigenstates and

$$
p_{n u l l}^{\mu}=\frac{d x^{\mu}}{d \lambda}
$$

is the tangent vector to the neutrino worldline $x^{\mu}$, parameterized by $\lambda$. From here we follow the Cardall and Fuller paper [26]. The covariant Dirac equation in curved space-time is (in natural units) [28]

$$
\left[i \gamma^{\mu}(x) D_{\mu}-m\right] \psi=0,
$$

where the matrices $\gamma^{\mu}(x)$ are related to the usual Dirac matrices $\gamma^{\hat{a}}$ by means of the vierbein fields $e_{\hat{a}}^{\mu}(x)$, i.e.

$$
\gamma^{\mu}(x)=e_{\hat{a}}^{\mu}(x) \gamma^{\hat{a}} .
$$

The Greek (Latin with hat) indices refer to curved (flat) space-time. $D_{\mu}$ is defined as

$$
D_{\mu}=\partial_{\mu}+\Gamma_{\mu}(x),
$$

where $\Gamma_{\mu}(x)$ are the spin connections

$$
\Gamma_{\mu}(x)=\frac{1}{8}\left[\gamma^{\hat{a}}, \gamma^{\hat{b}}\right] e_{\hat{a}}^{\nu} e_{\nu \hat{b} ; \mu},
$$

(semicolon represents the covariant derivative). Using the relation

$$
\gamma^{\hat{a}}\left[\gamma^{\hat{b}}, \gamma^{\hat{c}}\right]=2 \eta^{\hat{a} \hat{b}} \gamma^{\hat{c}}-2 \eta^{\hat{a} \hat{c}} \gamma^{\hat{b}}-2 i \epsilon^{\hat{d} \hat{a} \hat{b} \hat{c}} \gamma_{\hat{d}} \gamma^{5}
$$

where $\eta_{\hat{a} \hat{b}}$ is the metric of flat spacetime, and after some manipulations, the spin connections can be cast in the form [26]

$$
\gamma^{\mu} \Gamma_{\mu}=\gamma^{\hat{a}} e_{\hat{a}}^{\mu} \Gamma_{\mu}=\gamma^{\hat{a}} e_{\hat{a}}^{\mu}\left\{i A_{G \mu}\left[-(-g)^{-1 / 2} \gamma^{5}\right]\right\},
$$

where

$$
A_{G}^{\mu}=\frac{1}{4} \sqrt{-g} e_{\hat{a}}^{\mu} \varepsilon^{\hat{a} \hat{b} \hat{c} \hat{d}}\left(e_{\hat{b} \nu ; \sigma}-e_{\hat{b} \sigma ; \nu}\right) e_{\hat{c}}^{\nu} e_{\hat{d}}^{\sigma}
$$

and

$$
g=\operatorname{det}\left(g_{\mu \nu}\right)
$$

The above procedure allows to separate out the chirality operator $\gamma^{5}$. This shows that $\gamma^{\mu} \Gamma_{\mu}$ acts differently on left- and right-handed neutrino states. In fact, by writing

$$
\gamma^{5}=P_{R}-P_{L},
$$

where

$$
P_{L, R}=\frac{1 \mp \gamma^{5}}{2}
$$

are the projection operators, one sees that neutrinos with left- and right-handed helicity acquire a different gravitational contribution. In the case of neutrino oscillations, one can add, without physical consequences, a term proportional to the identity matrix $\left(\sim A_{G \mu} I\right)$, so that $\gamma^{5}$ can be replaced by the left-handed projection operator $\mathcal{P}_{L}=\left(1-\gamma^{5}\right) / 2$. As a consequence, the spin-gravity coupling is pushed in the lefthanded sector of the effective Hamiltonian (see Eqs. (6)-(8)), and no contributions appear in the right handed sector.

The equation of evolution of neutrinos has the form $(f=$ $\left.e, f^{\prime}=\mu, \tau\right)$

$$
i \frac{d}{d \lambda}\left(\begin{array}{c}
\nu_{f L} \\
\nu_{f^{\prime} L} \\
\nu_{f R} \\
\nu_{f^{\prime} R}
\end{array}\right)=\mathcal{H}\left(\begin{array}{c}
\nu_{f L} \\
\nu_{f^{\prime} L} \\
\nu_{f R} \\
\nu_{f^{\prime} R}
\end{array}\right),
$$

where the diagonal terms of the Hamiltonian $\mathcal{H}$

$$
\operatorname{diag} \mathcal{H}=U^{-1} M U+\Omega_{G}(x) \mathcal{P}_{L},
$$

are written in terms of the mixing matrix $U$ and mass matrix $M$

$$
M=\left(\begin{array}{cc}
m_{1}^{2} & 0 \\
0 & m_{2}^{2}
\end{array}\right),
$$

and $\Omega_{G}$ is defined as

$$
\Omega_{G}(x) \equiv \frac{p^{\mu} A_{G \mu}}{E} .
$$

$m_{1}$ and $m_{2}$ are the mass eigenstates, $\theta$ is the vacuum mixing angle, and $p^{\mu}=(E, \mathbf{p})$, being $E$ the energy measured in the local frame.

The inclusion of matter induced effective potential and magnetic terms, which appear in the off-diagonal terms of the Hamiltonian $\mathcal{H}$, will be discussed later. 


\subsection{The Geometry of a Rotating Mass Source}

For geometries with a spherical symmetry, such as the Schwarzschild or Reissner-Nordstrom space-times, $A_{G}^{\mu}$ vanish. Nevertheless, for rotating gravitational sources (LenseThirring geometry), whose line element is (in weak field approximation)

$$
d s^{2}=(1-\phi)(d t)^{2}-(1+\phi)(d \mathbf{x})^{2}-2 \mathbf{h} \cdot d \mathbf{x} d t,
$$

with

$$
\begin{aligned}
\mathbf{x} & =(x, y, z), \quad r=\sqrt{x^{2}+y^{2}+z^{2}}, \\
\phi(r) & =\frac{2 G M}{r}, \quad \mathbf{h}=\left(\frac{4 G M R^{2}}{5 r^{3}}\right) \boldsymbol{\omega} \wedge \mathbf{x},
\end{aligned}
$$

$\boldsymbol{\omega}$ is the angular velocity of the gravitational mass $M$, and $R$ its radius, $A_{G}^{\mu}$ acquire a non vanishing component related to the off-diagonal terms of the metric tensor,

$$
A_{G}^{\mu}(x)=\left(0,-\frac{4}{5} \frac{G M R^{2}}{r^{3}} \boldsymbol{\omega}^{\prime}\right),
$$

where

$$
\boldsymbol{\omega}^{\prime}=\boldsymbol{\omega}-\frac{3(\boldsymbol{\omega} \cdot \mathbf{x}) \mathbf{x}}{r^{2}} .
$$

One can show the angular velocity induces a drift velocity of neutrinos [29].

The vierbein fields used for computing the spin connection $A_{G}^{\mu}$ are

$$
\begin{gathered}
e_{0 \hat{0}}=(1+\phi), \quad e_{j \hat{i}}=-(1-\phi) \delta_{j i}, \\
e_{0 \hat{i}}=-h^{i}, \quad e_{i \hat{0}}=0 .
\end{gathered}
$$

The non vanishing $A_{G}^{\mu}$ is an indication of a preferred direction related to the angular velocity of the source. $\Omega_{G}(x)$ can be rewritten as

$$
\begin{aligned}
\Omega_{G}(r) & =\frac{p_{\mu} A_{G}^{\mu}(r)}{E_{l}}=\frac{4 G M R^{2}}{5 r^{3} E_{l}} \mathbf{p} \cdot \boldsymbol{\omega}^{\prime} \\
& \sim 10^{-13} \frac{M}{M_{\odot}}\left(\frac{R}{10 \mathrm{~km}}\right)^{2}\left(\frac{20 \mathrm{~km}}{r}\right)^{3} \frac{\omega^{\prime} \cos \beta}{10^{4} \mathrm{~Hz}} \mathrm{eV} .
\end{aligned}
$$

As the angular velocity goes to zero, the spherical symmetry is recovered and the spin connections vanish, as immediately follows from the above expressions.

It is worth note that the term $\gamma^{\mu} \Gamma_{\mu}$ can be rewritten in the form $(\sqrt{-g} \sim 1+2 \phi)$

$$
i \gamma^{\mu} \Gamma_{\mu} \simeq i \gamma^{\mu}\left(-i A_{G \mu} \gamma^{5}\right)=f(r) \gamma^{0} \omega^{\prime} \Sigma^{i}
$$

where

$$
f(r)=\frac{4 G M R^{2}}{5 r^{3}}
$$

and

$$
\Sigma^{i}=\gamma^{5} \gamma^{0} \gamma^{i}=\left(\begin{array}{cc}
\sigma^{i} & 0 \\
0 & \sigma^{i}
\end{array}\right)
$$

Here $\sigma^{i}, i=1,2,3$ are the Pauli matrices.

The term $\sim f(r) \boldsymbol{\omega}^{\prime} \cdot \boldsymbol{\Sigma}$ is the well known gravitomagnetic-spin coupling (see for example [30]).

\section{Matter Induced Effective Potential}

Neutrinos inside their neutrinospheres are trapped owing to weak interactions with the background matter, which lead to the potential energy

$$
V_{\nu_{f}} \simeq 3.8 \frac{\rho}{10^{14} \mathrm{gr} \mathrm{cm}^{-3}} y_{f}(r, t) \mathrm{eV},
$$

where $\rho=m_{n} n_{e}$ is the matter density, $m_{n}$ is the nucleon mass, $f=e, \mu, \tau$, and

$$
y_{e}=Y_{e}-1 / 3, \quad y_{\mu, \tau}=Y_{e}-1 .
$$

In these expressions, $Y_{e}$ is the electron fraction. In the present analysis we shall envisage those neutrinos for which matter induced effective potential for (electron) left-handed neutrinos such that $V_{\nu_{e}} \ll 1$. This follows in the regions where the electron fraction $Y_{e}$ assumes the value $\approx 1 / 3\left(y_{f} \ll 1\right)$ $[31,14,32]$. These regions are located at $r \sim 15 \mathrm{~km}$ (see the paper by Nunokawa, Peltoniemi, Rossi and Valle [32]).

\section{Magnetic Field Inside a Protostar}

Electroweak interactions of neutrinos with matter background play a central role on the neutrino emission during the core collapse of supernovae, and in general, on star cooling mechanism of magnetized medium. The neutrino energy spectrum in presence of strong magnetic fields is modified, depending on their flavors.

Protoneutron stars possess strong magnetic fields whose strength is $\gtrsim 10^{12}-10^{14} \mathrm{G}$. Such strong fields can be also found near the surface of supernovae [34], neutron stars [35], and magnetostars [36]. A fundamental feature of large magnetic fields in such astrophysical systems is related to their effects on neutrinos, which through charged and neutral current interactions, modify the dispersion relations of neutrinos.

In computing the effects of strong magnetic fields on neutrinos propagation, the following condition holds

$$
m_{e}^{2} \ll e B \ll M_{W}^{2},
$$

where $m_{e}$ is the electron mass and $M_{W}$ is the $W$-boson mass. We define the fields

$$
B_{c}=\frac{m_{e}^{2}}{e} \sim 4.4 \times 10^{13} \mathrm{G} \simeq 8.62 \times 10^{11} \mathrm{eV}^{2}
$$

It "separates" the regimes of weak field $B \ll B_{c}$, and of strong field $B \gtrsim B_{c}$.

In a series of recent papers [37, 38], a detailed analysis of the neutrinos physics in strong magnetic fields has been carried out. In what follows we shall consider those neutrinos which propagate orthogonally to the magnetic field of the protostar, so that $\mathbf{B} \cdot \hat{\mathbf{p}}=B \cos \alpha=0(\alpha=\pi / 2)$. The only contribution to the neutrino energy comes from the magnetic momentum of neutrinos. 


\subsection{Neutrino Magnetic Momentum}

Since neutrinos are uncharged particles, they do not interact directly with photons (magnetic fields). The typical coupling of the electromagnetic field with the fermionic current is absent for neutrinos. This is essentially due to the fact that the Standard Model is built up assuming that neutrinos are massless, hence only left-handed neutrinos appear in the theory. The experimental evidence of neutrino oscillations is an index that neutrinos are massive particles. In such a circumstance, we are in the context of a theory which goes beyond the Standard Model since right-handed projection of neutrinos has to be included in the fermion sector. As a consequence, an anomalous magnetic momentum emerges through quantum corrections (one-loop diagram whose internal lines are charged lepton and $W-Z$ boson propagators). Thus, even though neutrinos have no charged, they posses a magnetic momentum, which induces the interaction with photons [41]

$$
\hat{\mu}_{\nu}=\mu_{f f^{\prime}}=\frac{3 e G_{F} m_{\nu}}{8 \sqrt{2 \pi^{2}}} \sim 10^{-19} \mu_{B} \frac{m_{\nu}}{\mathrm{eV}}
$$

where $m_{\nu}$ is the neutrino mass, and

$$
\mu_{B}=\frac{e}{2 m_{e}}
$$

is the Bohr magneton.

Neutrinos interacting with the magnetic field of the protoneutron star acquire, hence, an energy through the interaction [39]

$$
\mathcal{L}_{i n t}=\bar{\psi} \hat{\mu}_{\nu} \sigma^{\hat{a} \hat{b}} F_{\hat{a} \hat{b}} \psi
$$

where $F_{\hat{a} \hat{b}}$ is the electro-magnetic field tensor, and

$$
\sigma^{\hat{a} \hat{b}}=\frac{1}{4}\left[\gamma^{\hat{a}}, \gamma^{\hat{b}}\right]
$$

In this paper, we shall consider the neutrino magnetic momentum

$$
\mu_{f f^{\prime}} \sim\left(10^{-12}-10^{-11}\right) \mu_{B},
$$

as provided by astrophysical and cosmological constraints [40]. Such a value on the neutrino magnetic momentum is great as compared with ones of the Standard Model prediction $\mu_{f f^{\prime}} \sim 10^{-19} \mu_{B}$, provided that the neutrino mass is $m_{\nu} \sim$ few eV [41].

The expression for the neutrino magnetic energy is

$$
\mu_{f f^{\prime}} B \sim 2.5 \times 10^{-6} \frac{\mu_{f f^{\prime}}}{10^{-11} \mu_{B}} \frac{B}{B_{c}} \mathrm{eV} .
$$

\section{Equation of evolution of neutrinos and the Asymmetric Neutrino Emis- sion}

Taking into account for the gravitational and magnetic contributions, the equation of evolution describing the conversion between two neutrino flavors $f=e$ and $f^{\prime}=\mu, \tau$ reads [42]

$$
i \frac{d}{d \lambda}\left(\begin{array}{c}
\nu_{f L} \\
\nu_{f^{\prime} L} \\
\nu_{f R} \\
\nu_{f^{\prime} R}
\end{array}\right)=\mathcal{H}\left(\begin{array}{c}
\nu_{f L} \\
\nu_{f^{\prime} L} \\
\nu_{f R} \\
\nu_{f^{\prime} R}
\end{array}\right),
$$

where, in the chiral base, the matrix $\mathcal{H}$ is the effective Hamiltonian defined as

$$
\begin{gathered}
\mathcal{H}=\left[\begin{array}{cc}
\mathcal{H}_{L} & \mathcal{H}_{f f^{\prime}}^{*} \\
\mathcal{H}_{f f^{\prime}} & \mathcal{H}_{R}
\end{array}\right], \\
\mathcal{H}_{L}=\left[\begin{array}{cc}
V_{\nu_{e}}+\Omega_{G}-\delta c_{2} & \delta s_{2} \\
\delta s_{2} & V_{\nu_{f^{\prime}}}+\Omega_{G}+\delta c_{2}
\end{array}\right], \\
\mathcal{H}_{R}=\left[\begin{array}{cc}
-\delta c_{2} & \delta s_{2} \\
\delta s_{2} & \delta c_{2}
\end{array}\right], \\
\mathcal{H}_{f f^{\prime}}=B_{\perp}\left[\begin{array}{cc}
\mu_{f f} & \mu_{f f^{\prime}} \\
\mu_{f f^{\prime}} & \mu_{f^{\prime} f^{\prime}}
\end{array}\right] . \\
\delta=\frac{\Delta m^{2}}{4 E}, \quad \Delta m^{2}=m_{2}^{2}-m_{1}^{2}, \\
c_{2}=\cos 2 \theta, \quad s=\sin 2 \theta,
\end{gathered}
$$

$B_{\perp}=B \sin \alpha=B$ is the component of the magnetic field orthogonal to the neutrino momentum, and $\beta$ is the angle between the neutrino momentum and the angular velocity.

$\Omega_{G}$ is diagonal in spin space, so that it cannot induce spinflips $^{1}$. Its relevance comes from the fact that it modifies the resonance conditions of spin-flips

$$
\begin{aligned}
& \nu_{f L} \rightarrow \nu_{f^{\prime} R} \quad V_{\nu_{e}}+\Omega_{G}(\bar{r})-2 \delta c_{2}=0, \\
& \nu_{f^{\prime} L} \rightarrow \nu_{f R} \quad V_{\nu_{f^{\prime}}}+\Omega_{G}(\bar{r})+2 \delta c_{2}=0 .
\end{aligned}
$$

$\bar{r}$ is the radial distance where the resonance occurs. The resonances (9) and (10) do not occur simultaneously; in what follows we shall consider the transition (9).

Besides, we shall use the best fit for solar neutrinos [43]

$$
\Delta m_{\text {Sun }}^{2} \sim\left(10^{-5} \div 10^{-4}\right) \mathrm{eV}^{2}, \quad \sin ^{2} 2 \theta_{\text {Sun }} \gtrsim 0.8
$$

$\Omega_{G}$ in (9) (and (10)) distorts the surface of resonance due to the relative orientation of the neutrino momentum with respect to the angular velocity. As a consequence, the outgoing energy flux $\mathbf{F}$ results modified. The neutrino momentum asymmetry is defined as [11]

$$
\frac{\Delta p}{p}=\frac{1}{3} \frac{\int_{0}^{\pi} \mathbf{F} \cdot \hat{\boldsymbol{\omega}} d a}{\int_{0}^{\pi} \mathbf{F} \cdot \hat{\mathbf{n}} d a},
$$

where the factor $1 / 3$ accounts for the conversion of neutrinos $\nu_{f L}$ into $\nu_{f^{\prime} R}\left(f \neq f^{\prime}\right), d a$ is the element of area on the distorted surface, $\hat{\boldsymbol{\omega}}$ and $\hat{\mathbf{n}}$ are the unit vectors parallel to the angular velocity and orthogonal to $d a$, respectively. To compute the fractional asymmetry one then should specify the protostar model [44], including into the hydrodynamical equations

\footnotetext{
${ }^{1}$ Of course $\Omega_{G}$ does not induce neutrino oscillations, unless one does not assume a violation of the equivalence principle, as in Refs [53].
} 
the rotational effects due to gravitational sources [45]. This task goes beyond the aim of this work.

Here we give an estimation of the fractional asymmetry following the calculations as in KS [2]. The surface of resonance is located to radial distance $r(\beta)=\bar{r}+\varrho \cos \beta$ $\left(\cos \beta=\hat{\mathbf{p}} \cdot \hat{\boldsymbol{\omega}}^{\prime}\right.$, with $\left.\hat{\boldsymbol{\omega}}^{\prime}=\boldsymbol{\omega}^{\prime} / \omega^{\prime}\right)$. Inserted in the resonance equation, one has

$$
\varrho=-\frac{f(r)}{V_{\nu_{e}}^{\prime}+\Omega_{G}^{\prime}} \simeq-\frac{f(r)}{V_{\nu_{e}}^{\prime}},
$$

where we used the resonance condition

$$
2 \delta c_{2}=V_{\nu_{e}}(r)
$$

and $\Omega_{G}^{\prime} \ll V_{\nu e}^{\prime}$ as evaluated at $\bar{r}$. The prime means derivative with respect to $r$. In such a calculations we assumed that the matter density profile is described by [46]

$$
\rho(r)=\rho_{0}\left(\frac{15 \mathrm{~km}}{r}\right)^{p},
$$

where $\rho_{0}=210^{14} \mathrm{gr} / \mathrm{cm}^{3}$ and $p=\mathcal{O}(1)$. The fractional asymmetry reads

$$
\begin{aligned}
\frac{\Delta p}{p} & =\frac{2 \varrho}{3 T} \frac{d T}{d r}=\frac{2}{3} \frac{f(\bar{r})}{T}\left(\frac{d V_{\nu_{e}}}{d T}\right)^{-1} \\
& =82.5 \frac{\mathrm{MeV}^{3}}{T y_{e}} \frac{f(\bar{r})}{\mathrm{eV}}\left(\frac{d n_{e}}{d T}\right)^{-1} .
\end{aligned}
$$

Since the density number $n_{e}$ is related to the temperature $T$ via the Fermi distribution

$$
n_{e}=2 \int \frac{d^{3} p}{(2 \pi)^{3}} \frac{1}{e^{(p-\mu) / T}+1},
$$

where $\mu$ is the chemical potential, one obtains $d n_{e} / d T \sim$ $2 \eta T^{2} / 3$, where $\eta \equiv \mu / T \sim 5$ (see the paper by Qian [7]). Eq. (15) then becomes

$$
\frac{\Delta p}{p} \sim 0.01\left[4.510^{5}\left(\frac{3 \mathrm{MeV}}{T}\right)^{3} \frac{1}{\eta y_{e}} \frac{f(\bar{r})}{\mathrm{eV}}\right] .
$$

From the resonance condition (9) one obtains

$$
\Delta m^{2} \cos 2 \theta \simeq 7.610^{4} y_{e} \mathrm{eV}^{2},
$$

as the density $\rho \sim 10^{11} \mathrm{gr} / \mathrm{cm}^{3}$ at $\bar{r} \sim 15 \mathrm{~km}$. The value falls into the best fit $(11)$ provided $y_{e} \approx 10^{-9}$. One can then see that for typical values of pulsars

$$
R \gtrsim 15 \mathrm{~km}, \quad M \sim M_{\odot}, \quad T \sim 3 \mathrm{MeV},
$$

Eq. (17) implies that the fractional asymmetry $\sim 1 \%$ is recovered as

$$
\omega \sim \text { few } 10^{2} \mathrm{~Hz}
$$

which is, indeed, the typical angular velocity of pulsars. It is worth to point out that such a result does not depend on the strength of the magnetic field. The latter only enters in studying the adiabaticity of the level crossing.

\subsection{Adiabatic Conditions}

Besides the level crossing (9), it must be also adiabatic, i.e. the corresponding (adiabatic) parameter $\gamma$, which quantifies the magnitude of the off-diagonal elements with respect to the diagonal ones of (6) in the instantaneous eigenstates, must satisfy the condition $\gamma(\bar{r}) \gg 1$.

The precession length $L$ coming from the effective Hamiltonian (6) is given by [47]

$$
L=\frac{2 \pi}{\sqrt{\left(2 \mu_{f f^{\prime}} B_{\perp}\right)^{2}+\left(V_{\nu_{e}}+\Omega_{G}-2 \delta c_{2}\right)^{2}}}
$$

At the resonance, it reads

$$
L_{r e s}=L(\hat{r})=\frac{\pi}{\mu_{f f^{\prime}} B_{\perp}} \simeq 8 \pi \frac{10^{-11} \mu_{B}}{\mu_{f f^{\prime}}} \frac{B_{c}}{B_{\perp}} \mathrm{m},
$$

The resonant spin flavor precession has the following width

$$
\delta r=2 \Lambda \lambda
$$

where

$$
\Lambda=\left(\frac{\rho^{\prime}(\bar{r})}{\rho(\bar{r})}\right)^{-1}=\frac{V_{\nu_{e}}(\bar{r})}{V_{\nu_{e}}^{\prime}(\bar{r})}
$$

assuming

$$
y_{f}^{\prime}(t, t)=0, \quad\left(Y_{e}^{\prime} \ll n_{e}^{\prime} / n_{e}\right)
$$

and

$$
\lambda=2 \frac{l}{L_{\text {res }}}, \quad l=\frac{1}{2 \delta} .
$$

The spin flavor conversion is adiabatic provided

$$
\delta r \gg L_{r e s},
$$

which is equivalent to

$$
\gamma=\frac{\left(\mu_{f f^{\prime}} B\right)^{2}}{\delta\left|\rho^{\prime} / \rho\right|} \gg 1 .
$$

This condition can be immediately verified using the above profile for $\rho(r)$, and $\mu_{f f^{\prime}} \sim 10^{-11} \mu_{B}$ and the typical values for the magnetic fields $B \sim\left(10^{12}-10^{14}\right) \mathrm{G}$.

\subsection{Spin Flip Probability}

The conversion probability $P_{\nu_{f L} \rightarrow \nu_{f^{\prime} R}}$ that the neutrino left flips into neutrino right is

$$
P_{\nu_{f L} \rightarrow \nu_{f^{\prime} R}}=\frac{1}{2}-\left(\frac{1}{2}-P_{L Z}\right) \cos 2 \tilde{\theta}_{i} \cos 2 \tilde{\theta}_{f},
$$

where the Landau-Zener probability is given by

$$
P_{L Z}=e^{-\gamma \pi / 2},
$$

and the effective mixing angle $\tilde{\theta}$ is defined as $[47,48]$

$$
\tan 2 \tilde{\theta}(r)=\frac{2 \mu_{f f^{\prime}} B}{\Omega_{G}(r)+V_{\nu_{e}}-2 \delta c_{2}} .
$$


$\tilde{\theta}_{i}=\tilde{\theta}\left(r_{i}\right)$ refers to initial mixing angle at $r_{i}$ where neutrinos are produced (i.e. at the neutrinosphere), and $\tilde{\theta}_{f}=\tilde{\theta}\left(r_{f}\right)$ is the mixing angle at $r_{f}$ where the neutrino helicity flip probabilities are evaluated.

Finally, we note that the weak field approximation is fulfilled since

$$
\frac{4 G M R^{2}}{5 \bar{r}^{3}} \omega R \lesssim 10^{-2}
$$

as $\omega \lesssim 10^{4} \mathrm{~Hz}$, and that rotational effects are relevant during the time scale $t_{0} \lesssim 10 \mathrm{sec}$ ( $t_{0}$ is the time scale for the emission of the energy $\sim 0.5 \times 10^{53} \mathrm{erg}$ by each neutrinos degree of freedom with $p \sim 10 \mathrm{MeV}$ ) [33].

\section{Conclusions}

It has been suggested a mechanism for the generation of pulsar kicks which accounts for the magnetic and gravitational fields of a rotating nascent star. Owing to the relative orientation of neutrino momenta with respect to the direction of the angular velocity, the energy flux turns out to be generated anisotropically. This effect is related to spin-gravity coupling, which differs for neutrinos with opposite helicity (left- and right-handed helicity). The estimations on $\Delta m^{2}-\sin ^{2} \theta$ parameters agree with the best fit of the present data on neutrino oscillations.

Some conclusive remarks are in order:

- Results discussed in this contribution, as well as the papers [17, 18, 19], in which pulsar kicks are discussed in relation to gravitational waves, have been obtained in semiclassical approximation, i.e. the gravitational field is described by the classical field equations of General Relativity. It will be of interest to investigate within the framework of quantum theories of gravity.

- Results suggest a correlation between the motion of pulsars and their angular velocities. Such a connection seems to be corroborated by recent analysis and observations discussed in $[49,50]$.

- The mechanism here proposed is strictly related to the gravito-magnetic effect, an effect predicted by General Relativity [30], as well as by many metric theories [51]. Its origin is due to the mass-energy currents (moving or rotating matter contribute to the gravitational fields, in analogy to the magnetic field of moving charges or magnetic dipole). Experiments involving the technology of laser ranged satellites [52] are at the moment the favorite candidate to test gravitomagnetic effects.

In connections with the mechanism proposed in this paper, a direct evidence of the gravitomagnetic effect seems to be provided by pulsar kicks. Future investigations on the velocity distribution of pulsars will certainly allow to clarify this still open issue.

\section{Acknowledgments}

The author thanks the organizers, in particular HansThomas Elze, of the Second International Workshop - DICE04, From Decoherence and Emergent Classicality to Emergent Quantum Mechanics, September 1-5, 2004 - Piombino (Italy).

Many thanks also to A. Kusenko and J.F. Nieves for discussions. Research supported by PRIN 2003.

\section{References}

[1] A.G. Lyne and D.R. Lorimer, Nature 369, 127 (1994) J.M. Corder and D.F. Chernoff, Atrophys. J. 505, 315 (1998). B.M.S. Hansen and E.S. Phinney, Mon. Not. R. Astron. Soc. 291, 569 (1997).

C. Fryer, A. Burrows and W. Benz, Astrophys. J. 496, 333 (1998).

H.-Th. Janka and E. Muller, Astron. Astrophys. 290, 496 (1994).

[2] A. Kusenko and G. Segre', Phys. Rev. Lett. 77, 4872 (1996).

[3] J.F. Nieves and P.B. Pal, Phys. Rev. D 40, 1693 (1989). J.C. D'Olivo, J.F. Nieves and P.B. Pal, Phys. Rev. D 40, 3679 (1989); Phys. Rev. Lett. 64, 1088 (1990).

S. Esposito and G. Capone, Z. Phys. C 70, 55 (1996).

J.C. D’Olivo and J.F. Nieves, Phys. Lett. B 383, 87 (1996).

P. Elmfors, D. Grasso and G.G. Raffelt, Nucl. Phys. B 479, 3 (1996).

[4] A. Kusenko and G. Segre', Phys. Lett. B 396, 197 (1997). A. Kusenko, astro-ph/0409521.

[5] G.M. Fuller, A. Kusenko, I. Mocioiu and S. Pascoli, Phys. Rev. D 68, 103002 (2003).

[6] A. Kusenko and G. Segre', Phys.Rev. D 59, 061302 (1999).

[7] Y.-Z. Qian, Phys. Rev. Lett. 79, 2750 (1997).

[8] E.Kh. Akhmedov, A. Lanza and D.W. Sciama, Phys. Rev. D 56, 6117 (1997).

[9] D. Grasso, H. Nunokawa and J.W.F. Valle, Phys. Rev. Lett. 81, 2412 (1998).

[10] R. Horvat, Mod. Phys. Lett. A 13, 2379 (1998).

[11] M. Barkovich, J.C. D’Olivo, R. Montemayor and J.F. Zanella, Phys. Rev. D 66, 123005 (2002).

M. Barkovich, J.C. D'Olivo and R. Montemayor, Phys. Rev. D 70, 043005 (2004).

[12] M. Barkovich, H. Casini, J.C. D'Olivo and R. Montemayor, Phys. Lett. B 506, 20 (2001).

[13] E. Nardi and J.I. Zuluaga, Astrophys. J. 549, 1076 (2001).

[14] M.B. Voloshin, Phys. Lett. B 209, 360 (1988); JEPT Lett. 47, 501 (1988)

[15] N.N. Chugai, Pis'ma A Zh. (Sov. Lett. Astron. J.) 10, 210 (1984).

[16] O.F. Dorofeef, V.N. Rodionov and I.M. Ternov, JEPT Lett. 40, 917 (1984).

[17] A. Burrows and J. Hayes, Phys. Rev. Lett. 76, 352 (1996). 
[18] H.J. Mosquera Cuesta, Astrophys. J. 544, L61 (2000); Phys. Rev. D 65, 061503 (2002).

H.J. Mosquera Cuesta and K. Fiuzza, Eur. Phys. J. C 35, 543 (2004).

[19] L.C. Loveridge, Phys. Rev D 69, 024008 (2004).

[20] A. Goyal, Phys. Rev. D 59, 101301 (1999).

D. Chandra, A. Goyal and K. Goswami, Phys. Rev. D 65, 053003 (2002).

[21] D. Lai, Y.-Z. Qian, astro-ph/9802345.

[22] J.R. Gott, J.E. Gunn and J.P. Ostriker, Astrophys. J. Lett. 201, L91 (1970).

E.R. Harrison and E.P. Tademaru, Astrophys. J. 201, 447 (1975).

[23] H.-Th. Janka and G.G. Raffelt, Phys. Rev. D 59, 023005 (1998).

[24] G. Lambiase, Pulsar Kicks Induced by Spin Flavor Oscillations of Neutrinos in Gravitational Fields, astro-ph/0411242. In this paper is studied the possibility that spin gravity coupling may induce a kick orthogonal (as well as parallel) to the angular velocity of the pulsar, as inferred in the statistical analysis perfomed in [50]. Nevertheless, recent data seem to exclude the possibility that pulsar motion is directed along a direction orthogonal to the angular velocity.

[25] G. Lambiase, Phys. Rev. D 71, 065005 (2005).

[26] C.Y. Cardall and G.M. Fuller, Phys. Rev. D 55, 7960 (1997).

[27] D.V. Ahluwalia, C. Burgard, Gen. Rel. 28, 1161 (1996); Phys. Rev. D 57, 4724 (1997).

N. Formengo, C. Giunti, C.W. Kim and J. Song, Phys. Rev. D 56, 1895 (1997).

K. Konno and M. Kasai, Progr. Theor. Phys. 100, 1145 (1998). T. Bhattacharya, S. Habib and E. Mottola, Phys. Rev. D 59, 067301 (1999)

S. Capozziello and G. Lambiase, Mod. Phys. Lett. A 14, 2193 (1999).

J.G. Pereira and C.M. Zhang, Gen. Rel. Grav. 32, 1633 (2000). P.M. Alsing, J.C. Evans and K.K. Nandi, Gen. Rel. Grav. 33, 1459 (2001).

J. Wudka, Phys. Rev. D 64, 065009 (2001).

G. Lambiase, Europ. J. Phys. C 19, 553 (2001).

[28] S.W. Weinberg, Gravitation and Cosmology (Wiley, New York, 1972).

N.D. Birrel and P.C.W. Davies, Quantum Fields in Curved Space (Cambridge University Press, Cambridge, 1982).

[29] H. Casini and R. Montemayor, Phys. Rev. D 50, 7425 (1994).

[30] I. Ciufolini and J.A. Wheeler, Gravitation and Inertia (Princeton University Press, 1995), and references therein.

[31] S.W. Bruen, Astrophys. J. Suppl. 58, 771 (1985).

J.T. Peltoniemi, Astron. Astrophys. 254, 121 (1992).

O.L.G. Peres and A. Yu Smirnov, Nucl. Phys. B 599, 3 (2001).

H. Athar, J.T. Peltoniemi and A. Yu Smirnov, Phys. Rev. D 51, 6647 (1995).

J.A. Pons, S. Reddy, M. Prakash, J.M. Lattimer and J.A. Miralles, Astrophys. J. 513, 780 (1999).

G.C. McLaughlin, J.M. Fetter, A.B. Balantekin and G.M. Fuller, Phys. Rev. C 59, 2873 (1999); Astropart. Phys. 18, 433 (2003)

D.O. Caldwell, G.M. Fuller and Y.-Z. Qian, Phys. Rev. D 61, 123005 (2000)
[32] H. Nunokawa, J.T. Peltoniemi, A. Rossi and J.W.F. Valle, Phys. Rev. D 56, 1704 (1997)

[33] G.G. Raffelt, Stars as Laboratories for Fundamental Physics (University of Chicago Press, 1996).

[34] V. Ginzburg, High Energy Gamma Ray Astrophysics (NorthHolland, Amsterdam, 1981).

D. Bhattacharya and G. Srinivasan, in: X-ray Binaries, ed. by W.H.G. Lewin, J. van Paradijs and E.P.J. van der Heuvel (Cambridge Univ. Press, Cambridge, 1995), p. 495.

[35] I. Fushiki, E.H. Gudmundsson and C.J. Pethick, Astrophys. J. 342, 958 (1989)

T.A. Mihara et al., Nature (london) 346, 250 (1990).

G. Chanmugam, Annu. Rev. Astron. Astrophys. 30, 143 (1992).

[36] R.C. Duncan and C. Thompson, Astrophys. J. Lett. 392, L9 (1992).

B. Paczynski, Acta Astron. 42, 145 (1992).

V.V. Usov, Nature (London) 357, 472 (1992).

C. Thompson and R.C. Duncan, Astrophys. J. 473, 322 (1966).

C. Kouveliotou et al., Nature (London) 393, 235 (1998); Astrophys. J. Lett. 510, L115 (1999).

[37] E. Erdas and G. Feldman, Nucl. Phys. B 343, 597 (1990). K. Bhattacharya and P.B. Pal, hep-ph/0212118.

[38] E. Elizalde, E.J. Ferrer and V. de la Incera, Ann. Phys. 295, 33 (2002)

E. Elizalde, E.J. Ferrer and V. de la Incera, Phys Rev D 70, 043012 (2004).

E.J. Ferrer and V. de la Incera, hep-ph/0308017.

[39] L.B. Okun, Yad. Fiz. 44, 847 (1986) [Sov. J. Nucl. Phys. 44, 546 (1986)].

L.B. Okun, M.B. Voloshin and M.I. Vyotsky, ibid. 91, 754 (1986) [ibid. 44, 440 (1986)].

C.S. Lim and W.J. Marciano, Phys. Rev. D 37, 1368 (1988).

E. Akhmedov and M. Khlopov, Yad. Fiz. 47, 1079 (1988) [Sov. J. Nucl. Phys. 47, 689 (1986)].

[40] G.G. Raffelt, Phys. Rep. 320, 319 (1999). S. Pakvasa and J.W. F. Valle, hep-ph/0301061.

[41] B.W. Lee and R.E. Shrock, Phys. Rev. D 16, 1444 (1977). K. Fujikawa and R.E. Shrock, Phys. Rev. Lett. 45, 963 (1980).

[42] D.D. Piriz, M. Roy and J. Wudka, Phys. Rev. D 54, 1587 (1996). M. Brüggen, Phys. Rev. D 58, 083002 (1998).

[43] K. Eguchi et al., Phys. Rev. Lett. 90, 021802 (2003). S.M. Bilenky et al., Phys. Rep. 379, 69 (2003). M. Maltoni, astro-ph/0401042.

[44] P.J. Schinder and S.L. Shapiro, Astrophys. J. 259, 311 (1982).

[45] G. Lambiase, Spin-Gravity Coupling of Neutrinos in Rotating Gravitational Fields and the Origin of Pulsar Kicks, in preparation.

[46] G.G. Raffelt, Astrophys. J. 561, 890 (2001).

[47] H. Nunokawa, Y.-Z. Qian and G.M. Fuller, Phys. Rev. D 55, 3265 (1997).

[48] W.C. Haxton, Annu. Rev. Astron. Astrophys. 33, 459 (1995). 
[49] See D. Lai, D.F. Chernoff and J.M. Cordes, Astrophys. J. 549, 1111 (2001); D. Lai, astro-ph/0312542, and references therein. For a more detailed analysis related to pulsars, see the references:

Z. Arzoumanian, D.F. Chernoff and J.M. Cordes, Astrophys. J. 568, 289 (2002).

J.M. Cordes and D.F. Chernoff, Astrophys. J. 505, 315 (1998). D.R. Lorimer, M. Bailes and P.A. Harrison, Mon. Not Astron. Soc 289, 592 (1997).

E. De Donder and D. Vanbeveren, New Astronomy 4, 167 (1999).

B.M.S. Hansen and S. Phinney, Mon. Not. R. Astron. Soc. 291, 569 (1997).

M. Toscano et al., Mon. Not. R. Astron. Soc. 307, 925 (1999).
[50] N. Wex, V. Kalogera and M. Kramer, Astrophys. J. 528, 401 (2000).

[51] C.M. Will, Theory and Experiment in Gravitational Physics (Cambridge University Press, 1993).

C.M. Will, The Confrontation Between General Relativity and Experiment: a 1998 Update, gr-qc/9811036.

A. Camacho, Int. J. Mod. Phys. Lett. A 16, 8396 (2001).

A. Barros and C. Romero, Mod. Phys. Lett. A 31, 2117 (2003).

[52] I. Ciufolini, E. Pavlis, F. Chieppa, E. Fernandes-Vieira and J. Perez-Mercader, Science 279, 2100 (2002).

[53] M. Gasperini, Phys. Rev. D 38, 2635 (1988).

A. Halprin and C.N. Leung, Phys. Rev. Lett. 67, 1833 (1991). 